\title{
Hydroacoustic Source Characterisation of Centrifugal Pumps
}

\author{
Fabrice Carta, Stéphane Bolpaire, Jacques Charley and Guy Caignaert \\ Laboratoire de Mécanique de Lille, ENSAM 8 Bd. Louis XIV, F-59046 Lille, France
}

(Received 8 January 2001; accepted 11 January 2001)

\begin{abstract}
This paper presents a study of the hydroacoustic behaviour of radial flow pumps in the low frequency range. New data on 'source terms' that have been obtained on two different test rigs: the first is a pump operating with water (test rig DERAP), and the second is a pump operating with air (SHF impeller, test rig VENTIL). In both cases, the experimental methodology is based on the use of pressure measurements in various points of the suction and delivery pipes, and plane wave propagation is assumed. An intensimetry technique, based upon the control of the wave speed, has been performed to obtain the state vector at different cross-sections. With such a hypothesis, the hydroacoustic behaviour of a pump is defined using a two-port model. This paper focuses on the determination of the transfer matrix and source terms of pressure and flow-rate fluctuations, in the frequency domain, in various operating conditions.
\end{abstract}

\section{INTRODUCTION}

Pump manufacturers, as well as pump users, are more and more concerned with the effects of pressure and flow rate fluctuations within a pump and the associated system. These fluctuations are directly related to vibration and noise and so have direct consequences on the safety of a system and environmental effects. ${ }^{1}$

For pumps, transmission properties as well as noise generation have to be considered. Pumps, and especially radial flow pumps, mainly produce noise at harmonics of their rotation frequency, which means that phenomena of interest are mostly in the low frequency range. In the low frequency range, wavelengths are long compared to the cross-sectional dimensions of the flow passages. Thus the various components of a system can generally be considered as two-port systems. $^{2,3}$

Up to now, transmission and source properties of a rotodynamic pump can only be determined experimentally. Using a two-port model with a transfer matrix formulation, in the frequency domain, it is easily demonstrated that three independent tests at least must be carried out in order to obtain all the unknown quantities. It is so very important, from an economic point of view, to be able to limit the number and the duration of such tests.

The present paper presents some results that have been obtained for two single volute radial flow pumps. One has been tested in air (as if it was a ventilator or a fan) and the other one has been tested with water. Firstly, the results clearly demonstrate that, in the low frequency range, transfer matrices can be considered as independent of the operating conditions if no cavitation appears during the operation. The transfer matrix of a pump in the low frequency range can so be experimentally determined during tests without rotation of the impeller with the use of two independent tests, with, for example, two kinds of external excitation or different kinds of boundary conditions. Then a third test during the normal operation of the pump can be used in order to obtain the source terms.

\section{HYDROACOUSTIC MODEL OF A PUMP}

In the low frequency range, pressure and flow rate fluctuations are considered as identical in one cross-section of the flow passage. And for each frequency, the Fourier transforms $P$ and $Q$ of these pressure and flow rate fluctuations are linked through the hydroacoustic transfer matrix and the source terms. Instead of using flow rate fluctuations $Q$, we use the fluctuations of the flow velocity and we define the product $\rho c V$ with: $\rho$ is the fluid density, $c$ is the wave speed and $V$ is the Fourier transform of the flow velocity fluctuations.

With such a choice of physical variables, the relationship can be written as follows:

$$
\left(\begin{array}{c}
P \\
\rho c V
\end{array}\right)_{\text {outlet }}=\left[\begin{array}{ll}
m_{11} & m_{12} \\
m_{21} & m_{22}
\end{array}\right]\left(\begin{array}{c}
P \\
\rho c V
\end{array}\right)_{\text {inlet }}+\left(\begin{array}{c}
A \\
B
\end{array}\right)_{\text {source }}
$$

So, the four terms $m_{i j}$ of the hydroacoustic transfer matrix are non dimensional. It must be remembered that these terms are complex functions of the frequency $f$. Such formulations are quite well-known and can be found for example in references $^{\mathbf{2}, \mathbf{4}, 5}$. The source terms $A$ and $B$ are also complex functions of the frequency $f$. Some other kinds of formulations of that relationship have been proposed by other authors as mentioned in reference ${ }^{6}$.

Generally, two kinds of tests are made:

a) first two independent tests are made to obtain the transfer matrix; when doing these tests with the pump in operation, one has to use external sources with levels high enough in order to allow one to neglect the terms $A$ and $B$

b) secondly a third test is made with the pump in operation in order to determine $A$ and $B$.

During each test, it is necessary to determine the pressure and velocity fluctuations at the pump inlet and outlet. This has been done by using the two-microphone technique ${ }^{7}$ in the straight pipes at the pump inlet and outlet. Auto- and crosspower spectra are measured, with the same phase reference in 